\title{
PROPIEDADES MECÁNICAS DE COMPUESTOS DE MATRIZ CEMENTICIA REFORZADOS CON TEJIDOS PRETENSADOS (PFRCM) ${ }^{1}$
}

\author{
E. BERNAT ${ }^{2,3}$, L. MERCEDES ${ }^{1}$, L. GIL $^{2}$ \\ ${ }^{1}$ Laboratorio para la Innovación Tecnológica de Estructuras y Materiales (LITEM), \\ Universitat Politècnica de Catalunya, Terrassa, España \\ ${ }^{2}$ Departamento Resistencia de Materiales e Ingeniería Estructural, \\ Universitat Politècnica de Catalunya, Terrassa, España \\ ${ }^{3}$ Serra Húnter Fellow
}

\begin{abstract}
RESUMEN
La matriz cementicia reforzada con tejidos (FRCM) es un material de refuerzo compuesto que se usa para reforzar estructuras de mampostería y de hormigón de forma pasiva, debido al requisito de activación de fisuras. Se propone pretensar el tejido para superar esta limitación, aumentar la resistencia a la fisuración y obtener una respuesta más rígida. Con este objetivo, se realizaron más de 200 ensayos de tracción en especímenes FRCM para analizar la influencia de los tejidos pretensados. También se analizaron otras variables, como el sistema de fijación, la velocidad de ensayo, el tipo de matriz y el tipo de tejidos. Las evidencias llevan a la conclusión de que pretensar el tejido del FRCM es una forma eficaz de aumentar su resistencia a la fisuración (más del 30\%) y la rigidez a tracción.
\end{abstract}

Palabras clave: Experimental; ensayo a tracción; FRCM; pretensado.

\begin{abstract}
Fabric Reinforced Cementitious Matrix (FRCM) is a composite strengthening material used to strengthen masonry and concrete structures in a passive way due to the requirement of crack activation. Prestressing fabric is proposed to overcome this limitation, to increase the cracking strength and to obtain a stiffer response. With this aim, over 200 tensile tests on FRCM specimens were performed to analyse the influence of prestressing fabrics. Other variables, like fixation system, testing speed, matrix material and fabric material, were also discussed. Evidences lead to conclude that prestressing fabric of FRCM is an effective way to increase its tensile cracking strength (over 30\%) and tensile stiffness.
\end{abstract}

Keywords: Experimental, tensile test, FRCM, Pre-stressing.

\footnotetext{
${ }^{1}$ Este articulo ha sido presentado previamente en una revista indexada (Bernat-maso et al., 2018)
} 


\section{INTRODUCCIÓN}

La matrices cementicias reforzadas con tejidos (FRCM) son un material compuesto de matriz inorgánica desarrollado inicialmente con el objetivo de reforzar estructuras de concreto y mampostería. La mayoría de los autores (ver (Awani et al., 2017)) han informado sobre el excelente rendimiento de este material compuesto utilizado como refuerzo permitiendo aumentar la resistencia y deformación ultima. Sin embargo, la naturaleza pasiva de FRCM hace que sea necesario romper la matriz de mortero para alcanzar la contribución total del tejido de refuerzo. Además, este hecho provoca grandes deformaciones en las estructuras reforzadas, lo que podría establecer los límites de diseño en el campo de servicio en lugar de en el campo de resistencia final. Por lo tanto, asegurar la contribución colaborativa de la fibra y la matriz desde el comienzo del proceso de carga es esencial, para evitar la fisuración de la matriz y para aumentar la rigidez de la estructura reforzada.

En esta línea, la investigación presentada en este documento quiere dar un paso adelante en la mejora de este material compuesto con el tejido pretensando. Es un enfoque prometedor para cumplir el objetivo particular de garantizar la total colaboración mecánica entre los dos componentes del FRCM. El aumento de la carga de fisuración contribuirá a mejorar la durabilidad del elemento reforzado, mientras que el aumento de la rigidez de FRCM lo hará aún más adecuado para fortalecer estructuras de concreto. Además, el tejido pretensado abriría la puerta para prefabricar elementos delgados de FRCM con aplicaciones que van más allá del refuerzo de estructuras.

Sin embargo, la idea de pretensar las fibras continúas embebidas en una matriz cementicia no es completamente nueva para el conocimiento de los autores. Según registros, fue propuesto por primera vez en 2001 por (Krüger et al.,2001), donde analizaron la influencia de hilos de carbono y aramida pretensados embebidos en matrices cementicias en su resistencia a la extracción. Unos años después, en 2007, (Xu and Li, 2007) presentaron un estudio que analizó la influencia de varios parámetros en la resistencia de adherencia de la matriz y la fibra, evaluada mediante ensayo de extracción. Uno de estos parámetros fue el pretensado de la fibra. El mismo año, la investigación llevada a cabo por (Peled, 2007) estudió la influencia de los tejidos pretensados de baja tensión ( 7MPa) embebida en la matriz cementicia por medio de ensayos de flexión, ensayos de extracción y observaciones SEM combinadas con un ensayo visco-elástico, para concluir que los tejidos más rígidos con arrastre reducido son los que mejoran aún más el rendimiento de fisuración de los compuestos de FRCM.

Por lo tanto, hasta donde sabemos, la respuesta a la tracción de matrices cementicias reforzadas con tejidos pretensados (denominada PFRCM de ahora en adelante) ha sido poco estudiada. Por lo tanto, el objetivo principal de esta investigación es analizar el rendimiento de las muestras de PFRCM para confirmar la hipótesis de que las fibras de pretensadas contribuirían a aumentar la resistencia a la fisuración y el módulo elástico antes de la fisuración.

En la misma línea, se ha propuesto una notable diversidad de sistemas de sujeción de las muestras a la máquina de ensayo. Entre ellos, la sujeción con mordaza (ver (Escrig et al.,2015; Carozzi and Poggi,2015; Garmendia et al., 2015)) y la sujeción a través de horquilla (ver (RILEM Technical Committee 232-TDT (Wolfgang Brameshuber) et al., 2016)) son las más comunes. La influencia del sistema de sujeción ha sido ampliamente estudiada (ver (De Santis and De Felice, 2015; Arboleda et al., 2016)), concluyendo que los sistemas de sujeción con mordazas proporcionan una respuesta más estable y una mayor capacidad de carga, mientras que el proceso de deslizamiento de fibra-matriz solo puede evaluarse utilizando los sistemas de transmisión de carga tangencial con el sistema de sujeción a través de horquillas. (Carozzi and Poggi, 2015) también estudiaron esta influencia de los sistemas de fijación en el modo de fallo.

Por lo tanto, un objetivo secundario de la investigación actual fue analizar la influencia de la velocidad de ensayo y la sujeción de la muestra en la respuesta mecánica de diferentes sistemas de FRCM (mortero y tejido que van más allá de las combinaciones comerciales típicas prescritas a límites de investigación 
más amplios) de manera integral para respaldar la definición del procedimiento de ensayo más adecuado para la caracterización de FRCM y PFRCM a tracción.

\section{MATERIALES Y METODOS}

\subsection{Mortero}

Se usaron dos morteros. El primero (S) es un mortero de reparación estructural (clase R3 según (UNEEN 1504-3:2006.) que incluye fibras de vidrio cortas y humo de sílice. El segundo (A) es un mortero autonivelante que incluye fibras y aditivos orgánicos.

La resistencia a la flexión y la resistencia a la compresión de cada mortero se determinaron experimentalmente de acuerdo con la norma (UNE-EN 1015-11:2000/A1:2007.). Los valores resultantes, junto con su coeficiente de variación (entre paréntesis), se resumen en la Tabla 1.

Tabla 1. Propiedades de las matrices de mortero

\begin{tabular}{|c|c|c|c|c|c|}
\hline \multirow{2}{*}{ Mortero } & \multirow{2}{*}{ Lote } & \multicolumn{2}{|c|}{ Resistencia a flexión (MPa) } & \multicolumn{2}{|c|}{ Resistencia a compresión (MPa) } \\
\hline & & Individual & Promedio & Individual & Promedio \\
\hline \multirow{4}{*}{$\mathrm{S}$} & $\mathrm{S} 1$ & 6.77 & \multirow{4}{*}{$5.89(0.16)$} & 33.49 & \multirow{4}{*}{$36.29(0.16)$} \\
\hline & $\mathrm{S} 2$ & 6.58 & & 34.66 & \\
\hline & S3 & 5.25 & & 44.81 & \\
\hline & S4 & 4.96 & & 32.20 & \\
\hline \multirow{4}{*}{ A } & A1 & 7.69 & \multirow{4}{*}{$8.73(0.21)$} & 22.23 & \multirow{4}{*}{$29.24(0.25)$} \\
\hline & $\mathrm{A} 2$ & 6.92 & & 26.98 & \\
\hline & A3 & 11.01 & & 39.50 & \\
\hline & A4 & 9.29 & & 28.23 & \\
\hline
\end{tabular}

\subsection{Tejidos}

Se utilizaron dos tejidos: fibra de carbono (C) y fibra de basalto (B). Las propiedades de las mallas y las fibras constitutivas utilizadas se resumen en la Tabla 2.

El sistema de pretensado y la metodología correspondiente (aplicación manual de la carga con tensores y controlada con dos células de carga de $10 \mathrm{kN}$, consulte la Figura 1) se utilizaron como máquina de ensayo para determinar la resistencia a la tracción representativa de los tejidos.

Estos ensayos dieron como resultado una capacidad de carga máxima de $1400 \mathrm{~N}$ por pieza de tejido de fibra de basalto pretensado ( $50 \mathrm{~mm}$ de ancho) y $2200 \mathrm{~N}$ por carbono (el mismo ancho). Estos valores corresponden a una tensión máxima equivalente de 528MPa para tejidos de basalto y 936MPa para tejidos de carbono, lo que demuestra que la tensión máxima equivalente de un tejido es mucho más baja que la resistencia máxima de la fibra (resumida en la Tabla 2).

\subsection{Método de producción y lista de especímenes.}

Se implementaron dos métodos de producción diferentes: uno para los especímenes FRCM convencionales y otro para los especímenes FRCM pretensados (PFRCM). En total se produjeron 90 especímenes convencionales y 135 especímenes de PFRCM. Para cada prueba, se produjeron 15 
especímenes para ensayar 5 velocidades de carga con tres repeticiones por prueba. Las 225 muestras tenían las mismas dimensiones: $400 \mathrm{~mm}$ x $50 \mathrm{~mm}$ x $9 \mathrm{~mm}$. El ancho $(50 \mathrm{~mm})$ estaba limitado por el ancho de los agarres que se utilizarían en el sistema de ensayo con mordaza, por lo que era más escaso que el mínimo recomendado de $60 \mathrm{~mm}$ (ver [16]). La longitud total de $400 \mathrm{~mm}$ permitió una longitud libre central de $200 \mathrm{~mm}$. Finalmente, el grosor $(9 \mathrm{~mm})$ se ajustó a las recomendaciones (> $6 \mathrm{~mm})$.

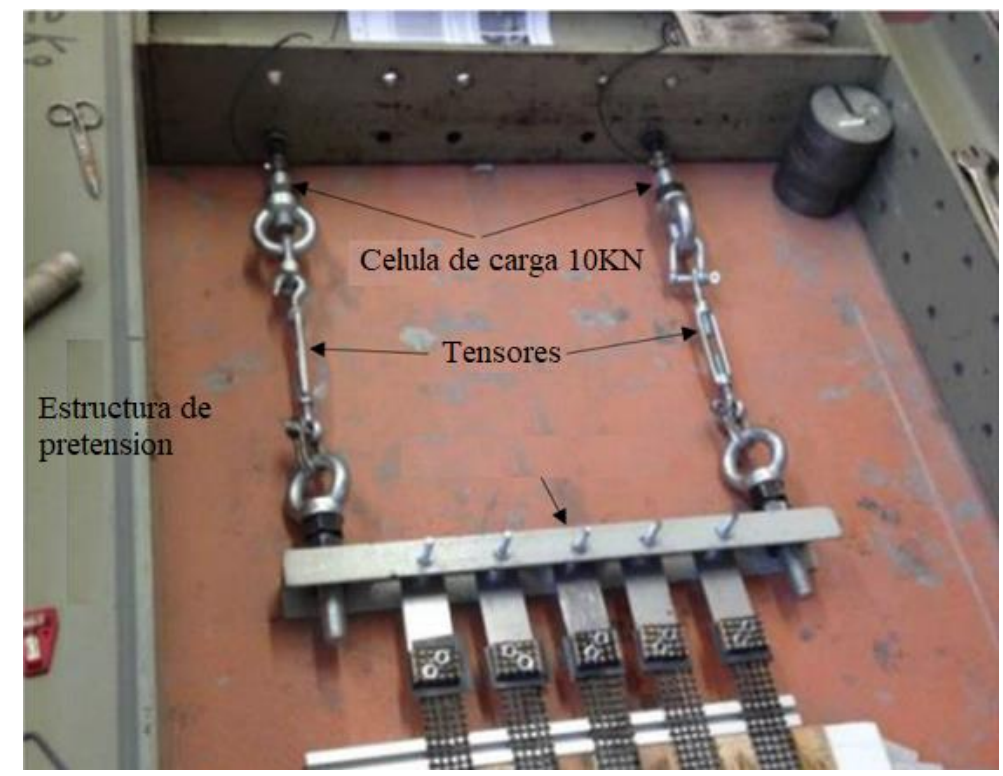

Figura 1. Tejidos al mecanismo de pretensado. Células de carga para controlar la carga de pretensado aplicada mediante tensores.

Tabla 2. Propiedades de las fibras y tejidos.

\begin{tabular}{|c|c|c|c|c|}
\hline & Propiedades & Unidades & Basalto (B) & Carbono $(C)$ \\
\hline \multirow{3}{*}{ Fibras } & Resistencia a tracción & $\mathrm{f}_{\mathrm{fib}, \mathrm{u}}[\mathrm{MPa}]$ & 3080 & 4320 \\
\hline & Modulo elástico & $\mathrm{E}_{\mathrm{fib}}[\mathrm{GPa}]$ & 95 & 240 \\
\hline & Deformación ultima & $\varepsilon_{\mathrm{fib}, \mathrm{u}}[\%]$ & 3.15 & 1.80 \\
\hline \multirow{6}{*}{ Tejidos } & Orientación de fibras & & Bidireccional & Bidireccional \\
\hline & Espesor equivalente & $\mathrm{t}_{\text {tex }}[\mathrm{mm}]$ & 0.053 & 0.047 \\
\hline & Recubrimiento & & No & No \\
\hline & Distancia entre mechones & $\mathrm{d}_{\text {tows }}[\mathrm{mm} \times \mathrm{mm}]$ & $15 \times 15$ & $10 \times 10$ \\
\hline & Módulo de elasticidad ${ }^{(1)}$ & $\mathrm{E}_{\text {tex }}[\mathrm{GPa}]$ & 63 & 113 \\
\hline & Resistencia a tracción ${ }^{(2)}$ & $\mathrm{f}_{\mathrm{fab}, \mathrm{u}}[\mathrm{MPa}]$ & 528 & 936 \\
\hline
\end{tabular}

(1) Valores de investigaciones anteriores [12]

(2) Fuerza representativa obtenida experimentalmente para el sistema de pretensado particular utilizado en esta investigación

La relación completa de los especímenes se presenta en la Tabla 3. Todas las muestras están etiquetadas con 3 letras, ABC, donde A corresponde al sistema de sujeción (C para sujeción con mordaza y H para la sujeción con horquilla), B representa el tipo de mortero (S para mortero de reparación y A para mortero autonivelante) y $\mathrm{C}$ son las siglas del tipo de tejido (C para carbono y B para basalto). En el caso de muestras pretensadas, se incluyeron dos letras adicionales antes del triplete anterior, lo que dio como resultado una etiqueta como: XY-ABC, donde XY representa el nivel de pretensado (LP para nivel de pretensado bajo, MP para nivel de pretensado intermedio y HP Para alto nivel de pretensado). Para analizar la influencia del sistema de sujeción, se compararon las muestras CSC/CSB y HSC/ HSB. El resto de especímenes fueron ensayados con el sistema de horquillas. Para cada combinación de 
mortero y tejido se produjeron muestras de PFRCM de carga baja pretensado (LP) y de alta carga de pretensado (HP). Finalmente, se probó un nivel de pretensado intermedio adicional para muestras hechas de mortero autonivelante y tejido de fibra de carbono (MP-HAC).

Tabla 3. Lista de especímenes producidos. Lote de mortero según Tabla 1.

\begin{tabular}{cccccc}
\hline $\begin{array}{c}\text { Tipo de } \\
\text { ensayo }\end{array}$ & $\begin{array}{c}\text { Lote de } \\
\text { mortero }\end{array}$ & Fibra & Fijación & $\begin{array}{c}\text { Nivel de } \\
\text { pretensado }\end{array}$ & $\begin{array}{c}\text { Numero de } \\
\text { especímenes }\end{array}$ \\
\hline CSC & S1 & C & C & - & 15 \\
\hline CSB & S1 & B & C & - & 15 \\
\hline HSC & S1 & C & H & - & 15 \\
\hline LP-HSC & S2 & C & H & $12.5 \%$ & 15 \\
\hline HP-HSC & S3 & C & H & $35 \%$ & 15 \\
\hline HSB & S1 & B & H & - & 15 \\
\hline LP-HSB & S2 & B & H & $12.5 \%$ & 15 \\
\hline HP-HSB & S4 & B & H & $25 \%$ & 15 \\
\hline HAC & A3 & C & H & - & 15 \\
\hline LP-HAC & A1 & C & H & $12.5 \%$ & 15 \\
\hline MP-HAC & A3 & C & H & $25 \%$ & 15 \\
\hline HP-HAC & A2 & C & H & $35 \%$ & 15 \\
\hline HAB & A3 & B & H & - & 15 \\
\hline LP-HAB & A4 & B & H & $12.5 \%$ & $25 \%$ \\
\hline HP_HAB & A3 & B & H & & \\
\hline
\end{tabular}

Las muestras de FRCM i PFRCM se prepararon según los detalles documentados en (Mercedes et al.,2018; Bernat-maso et al., 2018)

\subsection{Método de ensayo}

Las muestras ensayadas con el sistema de mordaza (CSC y CSB) se fijaron entre dos láminas de goma para evitar el contacto directo con las pinzas mecánicas (consulte la Figura 2.c), que podría haber introducido concentraciones de tensiones locales. No se observó deslizamiento relativo entre la muestra y la mordaza debido a la inclusión de láminas de goma. En este caso, la deformación se midió utilizando un extensómetro de distancia inicial de $50 \mathrm{~mm}$ con un rango de $25 \mathrm{~mm}$, que se colocó directamente en la posición central de las muestras fijadas (consulte la Figura 2.a).

En contraste, el sistema con horquilla incluía un grillete intermedio (ver Figura 2.d) para permitir todos los movimientos de rotación. Fue implementado para casi todos los ensayos reportados aquí. En este caso, el extensómetro se colocó en una herramienta de acero auxiliar conectada magnéticamente a los bordes internos de las placas de acero adherida a los especímenes. Eso permitió aumentar la longitud de referencia de medición hasta $200 \mathrm{~mm}$, pudiendo capturar la deformación promedio de toda la muestra (ver Figura 2.b y (Mercedes et al., 2018)).

Los ensayos de tracción se llevaron a cabo utilizando una prensa electromecánica MTS Insight con una capacidad de $10 \mathrm{kN}$. Para cada configuración de espécimen se realizaron 15 ensayos. Se incluyeron tres repeticiones para cada una de las cinco velocidades de ensayo diferentes: $\mathrm{V} 1=0.2 \mathrm{~mm} / \mathrm{min}, \mathrm{V} 2=1$ $\mathrm{mm} / \mathrm{min}, \mathrm{V} 3=5 \mathrm{~mm} / \mathrm{min}, \mathrm{V} 4=25 \mathrm{~mm} / \mathrm{min}$ y V5 $=100 \mathrm{~mm} / \mathrm{min}$. Las mediciones de fuerza y tensión se adquirieron automáticamente en una proporción de $50 \mathrm{~Hz}$, lo que proporcionó un promedio de 100 lecturas ( 2 segundos) para las pruebas más rápidas antes de la fisuración. 


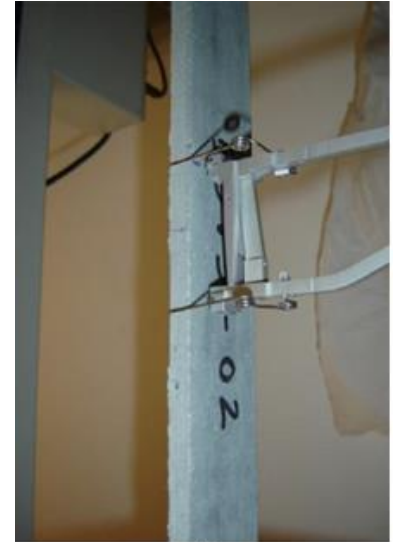

(a)

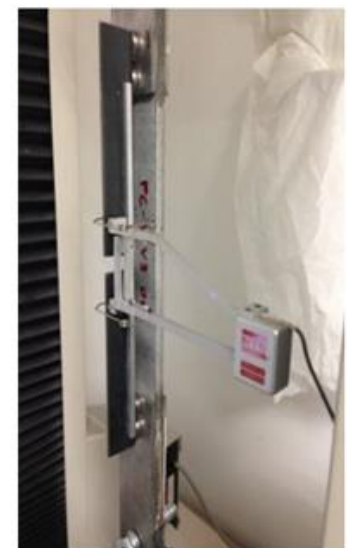

(b)

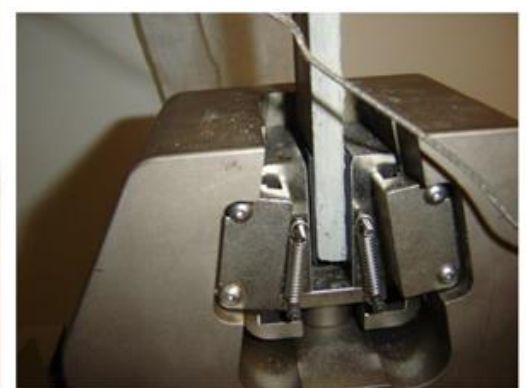

(c)

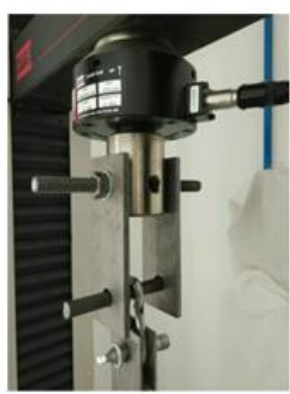

(d)

Figura 2. Posicionamiento del extensómetro para los ensayos con mordaza (a) y con horquilla (b). Sistemas con mordaza (c) y sistema con horquilla (d).

\section{RESULTADOS}

Las variables analizadas son la resistencia a la fisuración por tracción (fft), el módulo elástico efectivo antes de la fisuración $(\mathrm{Ef} *$ ), la resistencia máxima (ffu) y el módulo efectivo para la muestra fisurada (Ef), si las muestras no se rompen inmediatamente después de la fisuración.

La resistencia a la fisuración se calculó dividiendo la carga de fisuración por el área teórica de la fibra (ancho de la muestra $=50 \mathrm{~mm}$ x grosor equivalente, ttex). La resistencia final se calculó dividiendo la carga final por el área de fibra teórica.

Ef* se calculó como la pendiente de la curva de tensión-deformación antes de la carga de fisuración (ver Figura 5), mientras que Ef se calculó de acuerdo con (ACI 549.4R-13).

Se detectaron cuatro modos de fallo después de analizar los especímenes rotos. La correspondencia entre los modos de fallo y las tipologías de ensayos (materiales, nivel de pretensado y sistema de sujeción) que se resume en la Tabla 4. En la Figura 5 también se incluyen las imágenes de las fisuras múltiples típicas y la de una sola fisura, y la relación entre la velocidad de ensayo y el modo de fallo.

Los modos de fallo observados fueron: M-B: fisuración múltiple y rotura de la malla; M-S: fisuración múltiple y deslizamiento de la malla; O-B: Una fisura y rotura de malla; O-S: Una fisura y deslizamiento de malla. Los especímenes no pretensados están sombreados.

Los resultados obtenidos se presentan en la Figura 6 para los valores de resistencia y en la Figura 7 para el módulo de elasticidad y el módulo de fisuracion efectivo. Debe notarse que faltan la resistencia definitiva $\left(\mathrm{f}_{\mathrm{fu}}\right)$ y el módulo de fisuración efectivo (Ef) para algunos especímenes, ya que se rompieron inmediatamente después de que apareció la primera fisura (consulte la Tabla 4 con respecto a los modos de falla), por lo que la carga máxima para estos casos corresponde al de fisuración, pues ningún resultado significativo fue registrado después de la fisuración.

Con respecto a la variabilidad de los resultados, la Tabla 5 resume los coeficientes de variación promedio para cada propiedad analizada y la velocidad de prueba.

\section{DISCUSION}

\subsection{Influencia del sistema de sujeción.}

Primero, se observa que la influencia del sistema de sujeción en el modo de fallo desarrollado (ver Tabla 4). Por lo tanto, al comparar CSC con muestras de HSC y al comparar CSB con muestras de HSB, se observa que el sistema con mordaza (CSC y CSB) permitió el desarrollo de fisuración múltiple, mientras que las muestras ensayadas con sistema de horquillas (HSC y HSB) tendieron a desarrollar una sola fisura. Este hecho se debe al estado de tensión de compresión normal introducido por las 
mordazas, lo que reduce las posibilidades de deslizamiento de la fibra. En consecuencia, afectó el modo de fallo del tejido, que pasa de rotura (sistema con mordaza) a deslizamiento (sistema con horquillas) en términos generales. Por lo tanto, la posibilidad de deslizamiento del tejido, que está teóricamente asociada con el sistema de sujeción con horquillas, evita el desarrollo de múltiples fisuras. Por lo tanto, cuando la primera fisura se abre las fibras deslizan, y no se desarrollan más fisuras en la mayoría de los casos con sistema de horquilla.

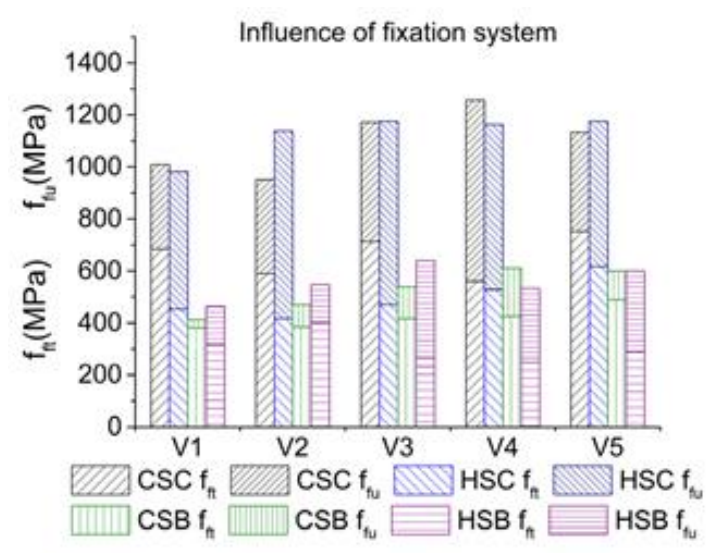

(a)

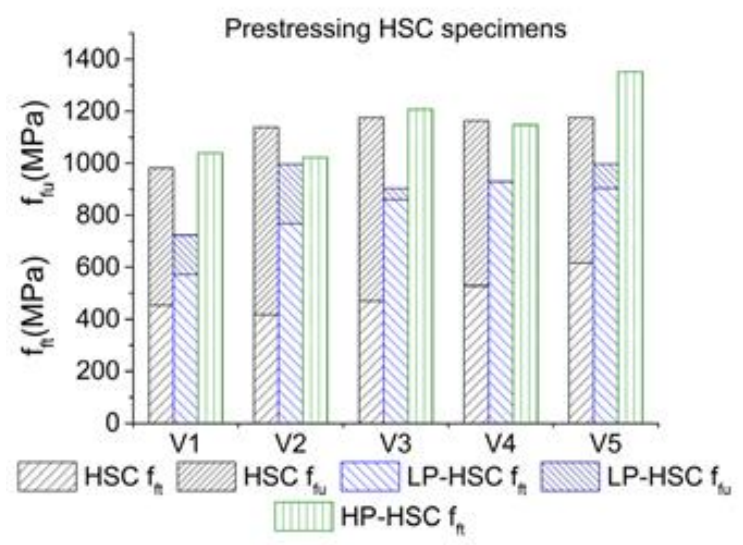

(c)

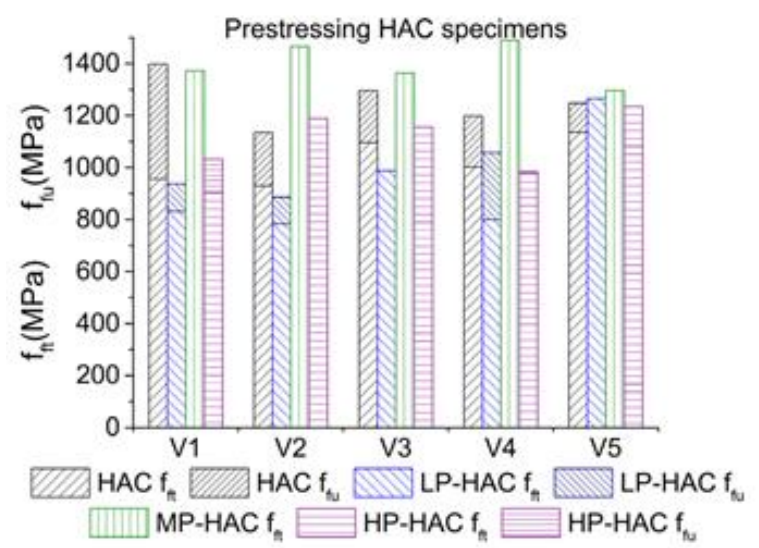

(e)

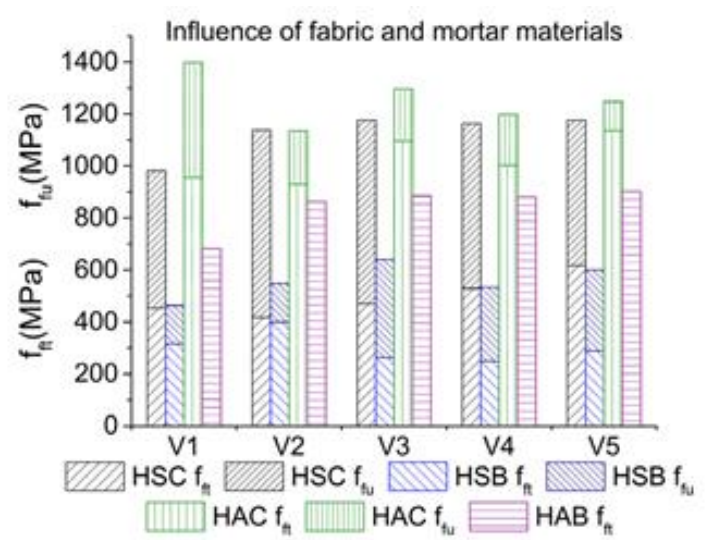

(b)

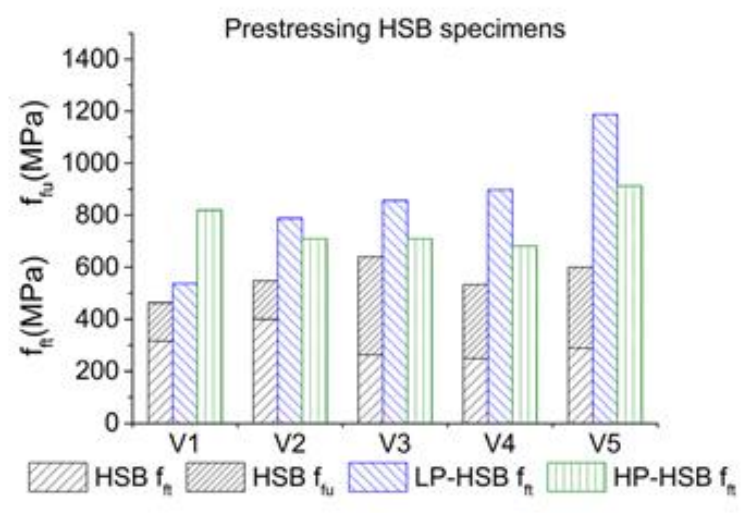

(d)

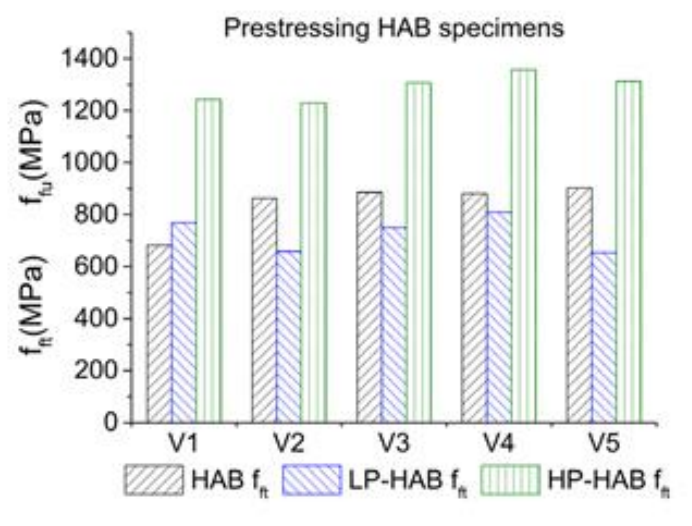

(f)

Figura 3. Resultados de fisuración $\left(\mathrm{f}_{\mathrm{ft}}\right)$ y resistencia máxima $\left(\mathrm{f}_{\mathrm{fu}}\right)$ para las cinco velocidades de prueba

(V1-V5). Etiquetado de especímenes según Tabla 3. 
Con respecto al análisis de resistencia (ver Figura 8.a), está claro que la carga de fisuración para las muestras ensayadas con el sistema de horquillas es menor que para las de sistema de mordaza. Esta evidencia apoya la idea anterior de que el estado de tensión de compresión normal introducido por las mordazas reduce las posibilidades de deslizamiento parcial de las fibras, lo que se traduce en mayores requisitos de carga para alcanzar la tensión de fisuración del material compuesto.

Con respecto a la influencia del método de sujeción en la resistencia final, no fue realmente significativo en los ensayos realizadas, ya que la mayoría de las muestras fallaron al romperse el tejido a un nivel de tensión similar.

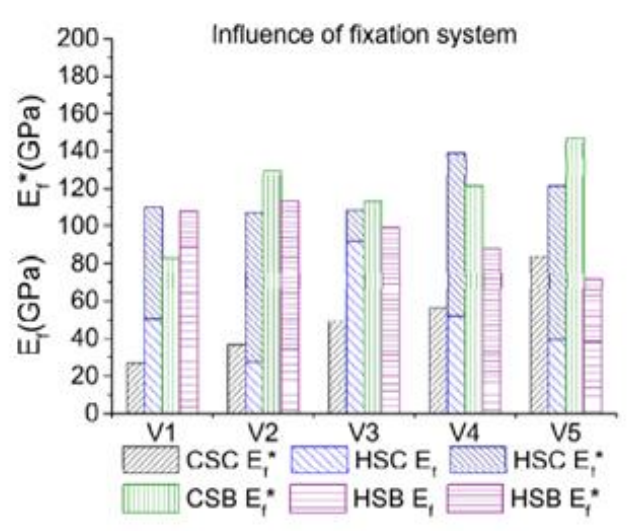

(a)

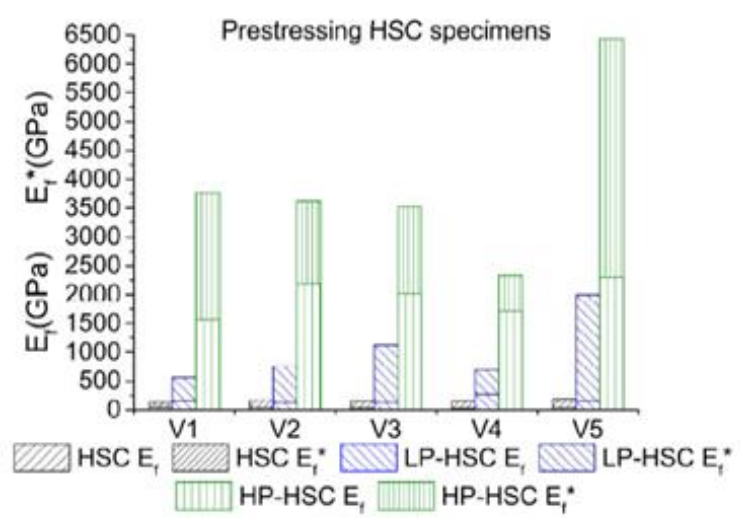

(c)

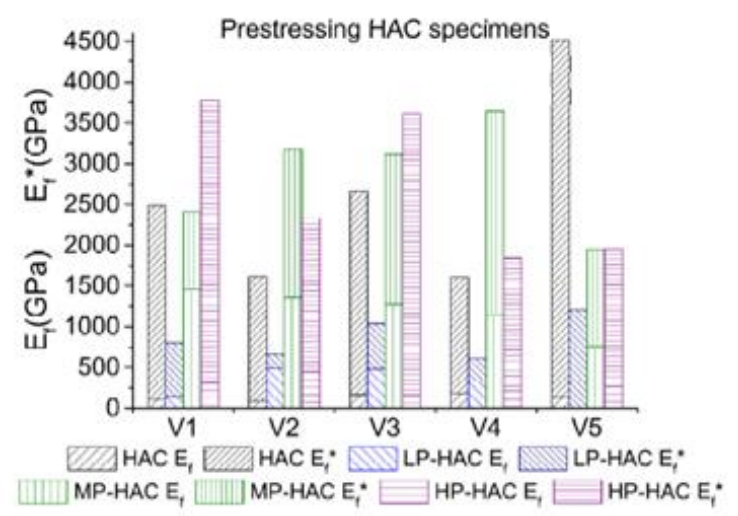

(e)

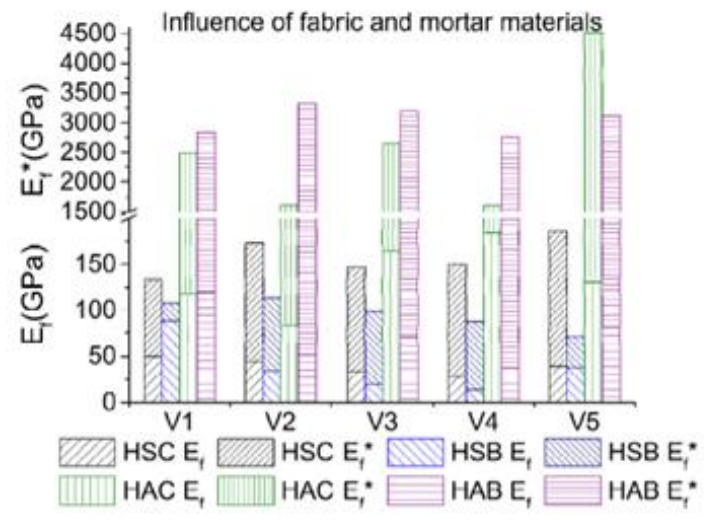

(b)

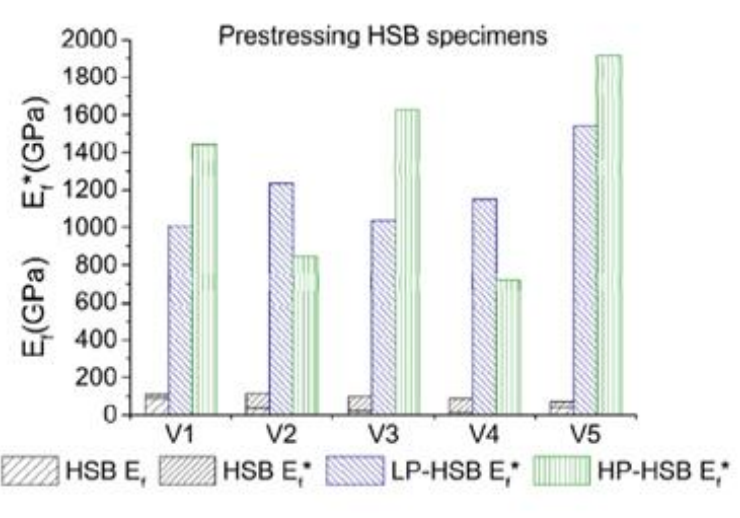

(d)

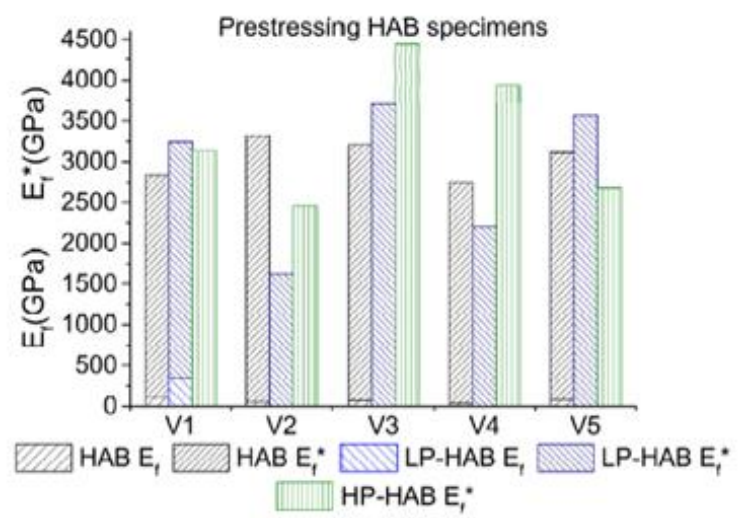

(f)

Figura 4. Módulo elástico antes de la fisuración (Ef*) y módulo de fisuración efectivo (Ef) para las cinco velocidades de ensayo (V1-V5). Etiquetado de especímenes según Tabla 3. 


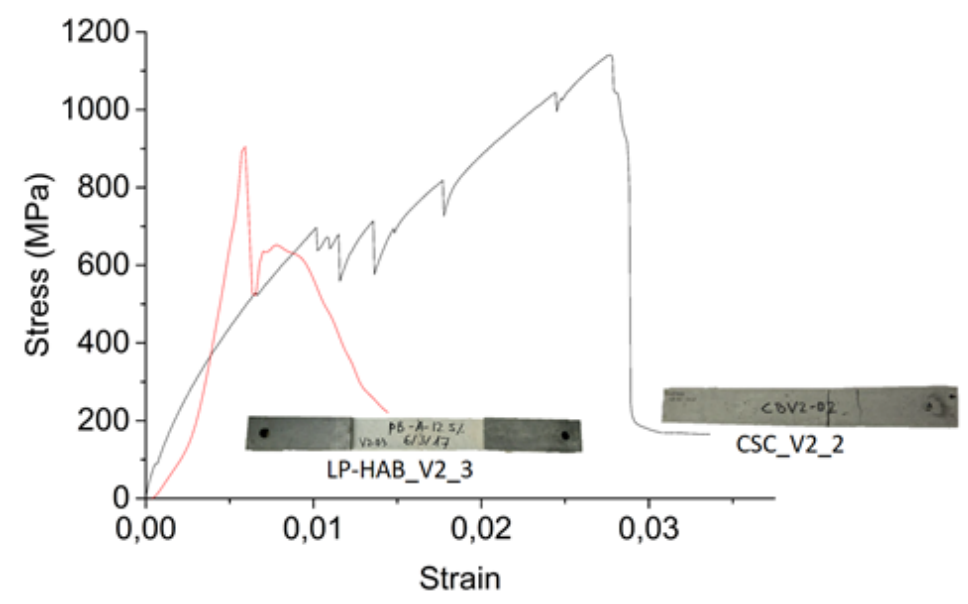

Figura 5. Curvas típicas de tensión-deformación para la fisuración multiple (CSC_V2_2) y una fisura y deslizamiento (LP-HAB_V2_3)

Tabla 4. Modo de falla principal para cada tipo de prueba dependiendo de la velocidad de ensayo.

\begin{tabular}{lllll}
\hline Tipo de espécimen & M-B & M-S & O-B & O-S \\
\hline CSC & V1-V5 & & & \\
\hline CSB & V3-V5 & & V1 & V2 \\
\hline HSC & V1-V2 & V3-V5 & & \\
\hline LP-HSC & & V1-V2 & & V3-V5 \\
\hline HP-HSC & & & & V1-V5 \\
\hline HSB & & & V1-V3 & V4-V5 \\
\hline LP-HSB & & & & V1-V5 \\
\hline HP-HSB & & & V1-V5 \\
\hline HAC & V1-V5 & \\
\hline LP-HAC & V1-V5 & \\
\hline MP-HAC & & & V1-V5 \\
\hline HP-HAC & V1 & V2-V5 \\
\hline HAB & & & V1-V5 \\
\hline LP-HAB & & & V1-V5 \\
\hline HP_HAB & & & V1-V5 \\
\hline
\end{tabular}

Tabla 5. Coeficiente de variación de las diferentes propiedades para cada velocidad de ensayo

\begin{tabular}{ccccc}
\hline \multirow{2}{*}{ Testing speed } & $\boldsymbol{f}_{\boldsymbol{f t}}$ & $\boldsymbol{E}_{\boldsymbol{f}}^{*}$ & $\boldsymbol{f}_{\boldsymbol{f u}}$ & $\boldsymbol{E}_{\boldsymbol{f}}$ \\
\cline { 2 - 5 } & $\boldsymbol{C o V}(\boldsymbol{\%})$ & $\boldsymbol{C o V}(\boldsymbol{\%})$ & $\boldsymbol{C o V}(\boldsymbol{\%})$ & $\boldsymbol{C o V}(\boldsymbol{\%})$ \\
\hline$V 1$ & 16.45 & 31.94 & 12.56 & 26.46 \\
\hline$V 2$ & 13.65 & 32.72 & 8.51 & 28.93 \\
\hline$V 3$ & 15.13 & 32.00 & 11.91 & 34.67 \\
\hline$V 4$ & 18.40 & 34.79 & 11.30 & 34.91 \\
\hline$V 5$ & 19.06 & 32.07 & 11.27 & 26.00 \\
\hline
\end{tabular}

Al observar la dependencia del módulo elástico (ver Figura 4a) del sistema de sujeción, se observa que el sistema con mordaza alcanzó un módulo elástico no fisurada más alto (Ef *) que el sistema con horquilla en el caso de muestras de tejido de basalto (CSB vs. HSB). Este efecto parece indicar que la adherencia entre el tejido de basalto usado y las matrices de mortero se vio realmente afectada por el 
sistema de sujeción. Además, esta tendencia no se observó para los especímenes de carbono, que pueden tener una mejor adherencia.

\subsection{Influencia del mortero.}

En la Figura 3 b se muestra que el aumento del rendimiento de tracción del mortero provoca un aumento directo de la resistencia a la fisuración del material compuesto. Por lo tanto, los fenómenos de fisuración están controlados principalmente por la matriz de mortero para muestras de FRCM sin pretensado. Además, la resistencia definitiva de las muestras con tejido de carbono también aumenta con el aumento del rendimiento de la resistencia a la tracción del mortero. Sin embargo, las muestras de HAB fallaron justo después de la fisuración debido a la resistencia relativamente baja de este tejido (1400N por muestra cómo se presentó anteriormente) en comparación con la alta resistencia a la tracción del mortero autonivelante usado (2200N por muestra). Por lo tanto, el tejido de basalto no pudo soportar la carga aplicada cuando la matriz se fisuro.

En la Figura 9b se observa que el módulo de elasticidad antes de la fisuración (Ef *) aumenta significativamente con el mayor rendimiento del mortero, mientras que el módulo de fisuración efectivo también se incrementa, pero se ve mucho menos afectado, lo que muestra que la influencia del mortero se nota principalmente durante la fisuración previa.

Finalmente, se observa (Tabla 4) que más muestras fallaron debido al deslizamiento del tejido cuando se usó el mortero autonivelante (A) en lugar del mortero de reparación (S). Este hecho puede estar relacionado con la adherencia tejido-mortero. Sin embargo, se necesita más investigación para confirmar esta declaración en particular.

\subsection{Influencia del tejido.}

La influencia del tipo de tejido se analiza comparando la respuesta de HSC con muestras de HSB y HAC con muestras de HAB respectivamente. Con respecto a la resistencia a la fisuración (ver Figura 6b), está claro que las muestras de tejido de carbono alcanzaron mayores esfuerzos antes de la fisuración que las de basalto. Esta respuesta se debe al mayor módulo elástico de los tejidos de carbono, que restringió la deformación en el mortero y requirió mayores cargas para romperla en comparación con el mortero reforzado con tejidos de basalto. De acuerdo con (De Santis and De Felice, 2015), este comportamiento es posible debido a un gran rendimiento mecánico de los tejidos usados en comparación con el bajo rendimiento de los morteros usados. En esta situación, la influencia del tejido en la primera fase elástica puede ser notable, mientras que no tiene ninguna influencia en aquellos casos que usaron morteros de alto rendimiento y tejidos relativamente flexibles.

La influencia del tipo de tejido en la resistencia a la tracción final también es notable: la resistencia final del HSC se duplicó para HSB, que fue del mismo orden de magnitud que la tensión de fisuración de las muestras de HSC. Este hecho apoya la idea de que la contribución de los tejidos de basalto es casi despreciable después de la fisuración. En relación con las muestras de mortero autonivelantes (HAC frente a HAB), se observó una tendencia similar: las muestras de HAC aumentaron la carga después de la fisuración y su resistencia máxima está en el rango de la de HSC, lo que respalda la idea de que el tejido controla el comportamiento posterior a la fisuración de la matriz. En contraste, las muestras de $\mathrm{HAB}$ fallaron en el momento de fisuración debido a la resistencia relativamente baja de ese tejido en particular.

El módulo de elasticidad (Figura 4b) antes de la fisuración es más alto para las muestras de HSC que para las muestras de HSB, lo cual era de esperar debido al mayor valor del módulo de elasticidad del tejido de carbono. Sin embargo, se registró la respuesta opuesta para los especímenes con mortero autonivelante. Por lo tanto, las muestras de HAC mostraron un módulo de elasticidad inicial más bajo que las muestras de HAB para 4 de las 5 velocidades de ensayo analizadas. Este hecho puede relacionarse con una mejor adherencia entre el mortero autonivelante y el tejido de basalto que entre el mismo mortero y el tejido de carbono. Después de la fisuracion, el módulo efectivo de fisuracion de 
HSC y HSB está en el mismo orden de magnitud y no se observa una influencia clara del tipo de tejido. En contraste, las muestras de HAC mostraron un módulo de fisuraciónn efectivo mayor que las muestras de $\mathrm{HAB}$, lo que representa claramente el hecho de que la capacidad de carga y la rigidez de las muestras de HAB se redujeron drásticamente después de la fisuracion.

Finalmente, el tipo de tejido influye en el modo de fallo (ver Tabla 4). Las muestras de tejidos de carbono tienden a mostrar fisuras múltiples en una proporción mucho mayor que los tejidos de basalto, donde el desarrollo de una sola fisura fue dominante entre los modos de fallo observados. Este hecho está relacionado con la ruptura o el deslizamiento del basalto inmediatamente después de que apareció la primera fisura, lo que limitó la posibilidad de desarrollar nuevas fisuras.

\subsection{Influencia de la carga de pretensado.}

El análisis de la influencia de la carga de pretensado debe tener en cuenta el lote de mortero particular utilizado para cada serie de muestras debido a la influencia significativa de este parámetro en la respuesta mecánica. Por lo tanto, las primeras muestras analizadas son aquellas producidas con el mismo lote de mortero. Comparando HAC con MP-HAC (Figura 6e) y HAB con HP-HAB (Figura 6f), está claro que el tejido pretensado contribuye a aumentar la resistencia a la fisuración $\left(\mathrm{f}_{\mathrm{ft}}\right)$ de manera significativa (un aumento promedio del $38 \%$ en el caso del tejido de carbono y $54 \%$ en el caso del tejido de basalto). Esto está justificado porque el tejido pretensado provoca un estado de tensión de precompresión en la matriz de mortero, que requiere que se fisuren a mayores niveles de cargas. Con respecto a la resistencia a la tracción $\left(\mathrm{f}_{\mathrm{fu}}\right)$, se debe tener en cuenta que las muestras de HAC sin pretensado continuaron aportando cargas más altas después de la fisuración, mientras que el MP-HAC falló en la carga de fisuración. En el caso de muestras de HAB, la tensión máxima correspondió a la de fisuracion para todos los casos.

El módulo elástico de deformación antes de la fisuración (Ef*) de las muestras MP-HAC pretensadas (Figura 9e) es más alto que las muestras HAC no pretensadas para las tres velocidades de ensayo intermedias y similares para la más lenta. De manera similar, el módulo elástico de deformación antes de la fisuración (Ef*) de las muestras de HP-HAB pretensadas (Figura 7f) es globalmente más alto que el módulo para las muestras sin pretensado (HAB), aunque la tendencia no es tan clara e incluso contradictoria para las velocidades de ensayo V2 y V5. En el caso del módulo efectivo de fisuración (Ef), se incrementa claramente al pretensar las muestras de HAC (comparando HAC y MP-HAC), mientras que no hay una comparación posible en el caso de las muestras de HAB cuyo modo de fallo típico incluido no resisten el aumento de carga después de fisurarse.

Finalmente, con respecto al modo de fallo (Tabla 4), se observa que MP-HAC tiende a fallar con el desarrollo de una sola fisura, mientras que las muestras de contraste sin pretensado (HAC) fallaron con fisuración múltiple.

Después de analizar los posibles casos de comparación directa, el resto de las muestras se analizan teniendo en cuenta la diversidad de los lotes de mortero utilizados. En el caso de muestras de HSC (ver Figura 6c y Figura 7c), el aumento de la carga de tensión previa contribuye a aumentar la resistencia a la fisuración, aunque la resistencia a la flexión del mortero mostró una reducción menor de S1 a S2 a S3 (ver Tabla 1). La resistencia a la tracción final de las muestras de HSC se redujo con la aplicación de un bajo nivel de pretensión y no se resistió ninguna carga adicional después de la fisuración en el caso de un nivel de pretensado alto. Por lo tanto, cuando la rotura de la fibra controla el fallo, el tejido pretensado reduce la resistencia máxima de FRCM, aunque puede mejorar la resistencia a la fisuración tanto como para superar la resistencia a la tracción final sin pretensado. Con respecto al módulo de elasticidad y al módulo efectivo de fisuración (ver Figura 7c), estos se incrementaron con la carga de tensión previa para las etapas de fisuración anteriores y posteriores, porque el tejido (el material más rígido del material compuesto) contribuyó más a la respuesta deformacional del FRCM. Sin embargo, el aumento sorprendentemente alto de los casos de HP-HSC debe analizarse teniendo en cuenta que el mortero S3 mostró más del 30\% de aumento en la resistencia a la compresión (ver Tabla 1). 
La misma tendencia de aumentar la resistencia a la fisuración con el nivel de tensión previa se observa en las muestras de HSB (Figura 6d), excepto en el caso de HP-HSB, que utilizó un mortero claramente más pobre que los otros dos casos de comparación. Estos casos también apoyan la idea de que los especímenes de FRCM pretensados tiende a causar la rotura en el punto de fisuración, aunque ocurre en tensiones más altas que la resistencia a la tracción final de los especímenes de comparación sin pretensado. En el caso de muestras de HSB (Figura 9d), el módulo elástico de la etapa de pretensado aumenta claramente con el nivel de pretensado, excepto en dos tipos de ensayos particulares que involucran las muestras de HP-HSB producidas con el lote de menor resistencia del mortero de reparación.

Al analizar todos los casos de HAC juntos (ver Figura 6e y Figura 7e), es evidente la dependencia de la resistencia a la fisuración por tracción de FRCM a la resistencia a la tracción del mortero (Tabla 1). El mortero A3 tenía una resistencia a la flexión más alta que A1 y esto fue mayor que para el lote de mortero A2. En consecuencia, la resistencia de fisuración de las muestras de HAC (mortero A3) fue mayor que para las muestras de LP-HAC (mortero A1) y el aumento de la resistencia de fisuración de LP-HAC a HP-HAC (mortero A2) fue más escaso de lo esperado debido a que las muestras de HPHAC que se produjeron con el mortero con menor resistencia a la flexión. Con respecto a la resistencia máxima a la tracción (ffu), la eficacia del tejido pretensado también se nota porque las muestras producidas con un mortero de menor resistencia a la flexión (HP-HAC) alcanzaron mayores cargas máximas, aunque en esos casos la carga máxima se registró en el momento de fisuración. En contraste, las muestras de LP-HAC soportaron una carga adicional después de la fisuración en varios casos. Se observa una tendencia similar para el módulo de elasticidad antes de la fisuración y el módulo efectivo de fisuración, que fueron más altos para las muestras de HP-HAC que para las muestras de LP-HAC, aunque las primeras usaron un mortero con menor resistencia a la flexión.

En el análisis HAB (ver Figura 6f y Figura 7f), el mismo razonamiento es posible. LP-HAB mostró una menor resistencia a la fisuración debido a que las muestras de LP-HAB se produjeron con un lote de mortero A4, que tuvo una resistencia a la flexión significativamente más baja que el lote A3 utilizado para las muestras de HAB. Respecto al módulo elástico antes de la fisuración, no se observó una tendencia clara.

En todos los casos, se ha observado (Tabla 4) que el aumento de la carga de pretensado tiende a estar relacionado con la limitación de la fisuración múltiple en favor del desarrollo de una sola fisura y también está relacionado con el aumento de los fallos por deslizamiento.

\subsection{Influencia de la velocidad de ensayo.}

Primero, no se observa una relación entre la velocidad de ensayo y los modos de falla. Además, tampoco es posible observar una clara dependencia de la velocidad de ensayo si se comparan casos individuales. La relación del valor medio (3 repeticiones) del valor promedio para la velocidad de prueba V1 se calcula para las velocidades de ensayo V2, V3, V4 y V5, para cada tipo de muestra y para cada parámetro (fft, ffu, Ef* y Ef) para definir un parámetro adimensional comparativo, $\mathrm{k}$. La idea es evaluar si los resultados para las velocidades de ensayo V2-V5 son mayores o menores que los correspondientes a las velocidades más lentas. Los resultados en comparación con el caso de referencia $(\mathrm{V} 1=0.2 \mathrm{~mm} /$ $\min$ ) fueron $\mathrm{k}=1.03, \mathrm{k}=1.17, \mathrm{k}=1.08 \mathrm{y} \mathrm{k}=1.25$ para las velocidades de ensayo V2, V3, V4 y V5 respectivamente.

Por lo tanto, teniendo en cuenta que la velocidad de ensayo V3 $(5 \mathrm{~mm} / \mathrm{min})$ alcanzó el máximo relativo del parámetro de comparación (k) y que esta velocidad se puede mantener constante incluso en los casos que muestran procesos deslizantes en un tiempo de ensayo totalmente razonable, se propone para utilizar $5 \mathrm{~mm} / \mathrm{min}$ como relación de deformación para ensayos de tracción FRCM. Sin embargo, se requieren futuras campañas de prueba adicionales para establecer o cambiar esta propuesta inicial, que es mucho más rápida que las tasas de prueba típicas utilizadas por otros investigadores, que tienden a estar en el rango de 0,1 mm/min a 0,5 mm/min (ver (Carozzi and Poggi, 2015; Arboleda et al., 2016)). 


\section{CONCLUSIONES}

Se ha realizado una campaña experimental que incluye 225 ensayos de tracción en especímenes de FRCM para analizar la influencia de los tejidos pretensado en las propiedades mecánicas de FRCM. Además, se ha estudiado la influencia del sistema de fijación, la velocidad de prueba, el mortero y el tejido.

Respecto a la técnica de pretensado, se puede concluir que:

- El pretensado del tejido del FRCM contribuye a aumentar la resistencia a la fisuración en más del $30 \%$ y a aumentar el módulo elástico antes la fisuración, aunque se observa una mayor variabilidad en este parámetro.

- El pretensado del tejido de FRCM cambia el modo de falla evitando el desarrollo de múltiples fisuras y favoreciendo el deslizamiento del tejido.

- El pretensado del tejido de FRCM causa una reducción de la resistencia máxima a la tracción en aquellos casos cuyo modo de falla es controlado por la rotura de la tracción de la fibra. Sin embargo, el aumento de la resistencia a la fisuración asociado con el proceso de pretensado puede sobrepasar la resistencia a la tracción final sin pretensado, lo que da como resultado que la carga de fisuración se convierta en la máxima.

- El pretensado es especialmente efectivo en aquellos casos en los que el modo de fallo está asociado con el deslizamiento del tejido.

Respecto al sistema de fijación, se puede concluir que:

- El sistema de fijación con mordazas tiende a desarrollar fisuras múltiples, mientras que el sistema de fijación con horquilla tiende a asociarse con desarrollo de una sola fisura debido a la mayor probabilidad de deslizamiento del tejido.

- Los ensayos con el sistema de mordaza obtuvieron valores más altos de resistencia a la fisuración y módulo de elasticidad no fisurado que los ensayos con el sistema de horquillas. Sin embargo, la resistencia a la tracción final no está influenciada por el sistema de fijación.

- El aumento de la resistencia a la flexión del mortero utilizado como matriz de FRCM provoca un aumento de la resistencia a la fisuración y el correspondiente módulo elástico antes de la fisuración. La influencia del mortero es mucho más significativa antes de la fisuración

- Las propiedades del tejido controlan la respuesta mecánica durante la etapa posterior a la fisuración, si la resistencia a la tracción del tejido es mayor que la matriz y la adherencia. El aumento de la rigidez del tejido contribuye a aumentar la rigidez de FRCM antes de la fisuración si existe una adherencia adecuada entre la matriz y el tejido.

- Se propone utilizar una velocidad de ensayo de $5 \mathrm{~mm} / \mathrm{min}$ porque está asociada con un máximo relativo del rendimiento de FRCM y permite utilizar una tasa de deformación constante incluso para los casos que se deslizan.

\section{AGRADECIMIENTOS}

Los autores agradecen el apoyo financiero del Ministerio de Economía y Competitividad del Gobierno de España (MINECO) y el Fondo Europeo de Desarrollo Regional (FEDER) a través del proyecto MULTIMAS (técnicas multiescala para el análisis experimental y numérico de la fiabilidad de las estructuras de mampostería, número de referencia BIA2015-63882-P). Se reconoce el apoyo brindado por los estudiantes de grado Albert Rosell, Daniel de la Fuente, Joan Antoni Vila, Joan Margens, Miquel Pérez y Sergi Fernández para realizar pruebas experimentales. También se reconoce la contribución del estudiante del máster internacional SAHC, Thomas Bose. 


\section{REFERENCIAS}

ACI Committee 549. (2013). ACI 549.4R-13 - Guide to design and construction of externally bonded fabric-reinforced cementitious matrix (FRCM) systems for repair and strengthening concrete and masonry structures. Farmington Hills: American Concrete Institute.

Arboleda, D., Carozzi, F. G., Nanni, A., \& Poggi, C. (2016). Testing Procedures for the Uniaxial Tensile Characterization of Fabric-Reinforced Cementitious Matrix Composites. Journal of Composites for Construction, 20(3), 4015063. https://doi.org/10.1061/(ASCE)CC.1943-5614.0000626 Awani, O., El-Maaddawy, T., \& Ismail, N. (2017). Fabric-reinforced cementitious matrix: A promising strengthening technique for concrete structures. Construction and Building Materials, 132, 94-111. https://doi.org/10.1016/j.conbuildmat.2016.11.125

Bernat-maso, E., Gil, L., Mercedes, L., \& Escrig, C. (2018). Mechanical properties of pre-stressed fabric-reinforced cementitious matrix composite ( PFRCM ). Construction and Building Materials, 191, 228-241. https://doi.org/10.1016/j.conbuildmat.2018.09.210

Carozzi, F. G., \& Poggi, C. (2015). Mechanical properties and debonding strength of Fabric Reinforced Cementitious Matrix (FRCM) systems for masonry strengthening. Composites Part B: Engineering, 70, 215-230. https://doi.org/10.1016/j.compositesb.2014.10.056

Committee AEN/CTN 83 (2006). UNE-EN 1504-3:2006. Products and systems for the protection and repair of concrete structures - Definitions, requirements, quality control and evaluation of conformity - Part 3: Structural and non-structural repair. Madrid: AENOR.

Committee AEN/CTN 83 (2007). UNE-EN 1015-11:2000/A1:2007. Métodos de ensayo de los morteros para albañilería. Parte 11: Determinación de la resistencia a flexión y a compresión del mortero endurecido. Madrid: AENOR.

De Santis, S., \& De Felice, G. (2015). Tensile behaviour of mortar-based composites for externally bonded reinforcement systems. Composites Part B: Engineering, 68, 401-413. https://doi.org/10.1016/j.compositesb.2014.09.011

Escrig, C. (2015). Estudio del comportamiento mecánico de vigas de hormigón armado reforzadas a flexión y a cortante con materiales compuestos de matriz cementítica, 416.

Garmendia, L., Larrinaga, P., San-Mateos, R., \& San-Jos??, J. T. (2015). Strengthening masonry vaults with organic and inorganic composites: An experimental approach. Materials and Design, 85, 102114. https://doi.org/10.1016/j.matdes.2015.06.150

Krüger, M., Reinhardt, H.-W., \& Fichtlscherer, M. (2001). Bond behaviour of textile reinforcement in reinforced and prestressed concrete. Otto-Graf-Journal, 12, 33-50.

Mercedes, L., Gil, L., Materials, E. B.-M.-C. and B., \& 2018, undefined. (2018). Mechanical performance of vegetal fabric reinforced cementitious matrix (FRCM) composites. Elsevier, 175, 161173. https://doi.org/10.1016/j.conbuildmat.2018.04.171

Peled, A. (2007). Pre-tensioning of fabrics in cement-based composites. Cement and Concrete Research, 37(5), 805-813. https://doi.org/10.1016/j.cemconres.2007.02.010

RILEM Technical Committee 232-TDT (Wolfgang Brameshuber), Brameshuber, W., Hinzen, M., Dubey, A., Peled, A., Mobasher, B., ... Jesse, F. (2016). Recommendation of RILEM TC 232-TDT: test methods and design of textile reinforced concrete: Uniaxial tensile test: test method to determine the load bearing behavior of tensile specimens made of textile reinforced concrete. Materials and Structures/Materiaux et Constructions, 49(12), 4923-4927. https://doi.org/10.1617/s11527-016-0839-z $\mathrm{Xu}, \mathrm{S} .$, \& Li, H. (2007). Bond properties and experimental methods of textile reinforced concrete. Journal Wuhan University of Technology, Materials Science Edition, 22(3), 529-532. https://doi.org/10.1007/s11595-006-3529-9 